\title{
Tunable ultra-long random distributed feedback fiber laser
}

\begin{abstract}
A $72 \mathrm{~km}$ open-ended symmetrical tunable random distributed feedback fiber laser (RDB-FL) with different pumping schemes is presented in this study. The random distributed feedback was contributed by Rayleigh scattering in the single-mode fiber while distributed gain was provided by the effect of stimulated Raman scattering. The pumping schemes tested with the configuration was outward and inward pumping, where these would be backward and forward pumping in a non-symmetrical configuration of a fiber laser, respectively. The tuning range was also varied in conjunction with the different pumping schemes to determine the optimum performance. Random lasing in the RDB-FL was achieved by utilizing multiple scattering in the disordered gain medium to achieve resonance. With pump power limited to $1.5 \mathrm{~W}$, the best threshold was measured as low as $1.4 \mathrm{~W}$ while the highest total output power was at $8 \mathrm{~mW}$. In outward pumping configuration, the wavelengths that are within the maximum Raman gain (1555-1565 nm) show the best peak powers and total output power with a narrow linewidth, as low as $0.25 \mathrm{~nm}$.
\end{abstract}

Keyword: Fiber laser; Raman gain; Random distributed feedback fiber laser; Random laser; Rayleigh scattering; Stimulated Raman scattering 